\title{
How Can a Mobile Service Provider Reduce Costs with Software-Defined Networking?
}

\author{
Bram Naudts $^{1}$, Mario Kind ${ }^{2}$, Sofie Verbrugge ${ }^{1}$, Didier Colle $^{1}$ and Mario Pickavet $^{1}$ \\ ${ }^{1}$ Ghent University/iMinds, ${ }^{2}$ Deutsche Telekom AG
}

\begin{abstract}
Network architecture innovation has been driven by virtualization and centralization of network control based on software-defined networking (SDN) and by network functions moved to the cloud with network function virtualization (NFV). These two principles are considered as promising enablers to reduce costs and spur innovation. In the first part of the paper we argue that the evolved packet system (EPS) can be seen as a service function chain and an area in which SPs can capitalize on SDN and NFV capabilities. A multi-layer modular architecture for carrier networks based on SDN- and NFV principles is provided. In the second part of the paper we focus solely on SDN, we argue that service providers (SPs) can benefit from SDN to reach cost savings in their mobile network. Our work focuses on the cost savings that can be reached via the centralization of control and the operational paradigm of developing and operating the network management software in-house instead of buying a vendor solution only. We quantify the potential cost savings that can be reached in an Internet protocol/multi-protocol label switching (IP/MPLS)-based transport service with SDN capabilities that interconnects the key functional elements in a mobile network. In the analysis, we evaluate the impact on capital expenditures (CapEx) and provide details on the impact on operational expenditures (OpEx). The evaluation can serve as a blueprint for techno-economic analysis of the mobile network operator's processes in the transport network of a mobile network
\end{abstract}

\section{INTRODUCTION}

The carrier market is traditionally standard driven. Network architectures, such as those introduced by the Third Generation Partnership Project (3GPP) undergo years of standardization and interoperability testing. These architectures are closed systems that were designed with their unique purpose in mind and are composed of many interfaces and components, each with their own definition or functions. Once standardized, each vendor has to implement the resulting standard in network equipment. As such, the implementation is vendor specific and the configuration interfaces vary between vendors and even between different products of the same vendor. As a result, operators often end up in a situation in which they need to purchase equipment from the same vendor to reach maximum efficiency. Due to vendor lock-in, carrier networks rely on pricy, vendor specific hardware platforms that run complex, distributed control software which is typically closed source and proprietary. However, as customer demand evolves and new technology emerges, the complex nature of these architectures starts to become a hindrance to sustainable growth. First, CapEx and OpEx will increase while at the same time the average revenue per user (ARPU) is flat or decreasing. Second, with profitability under pressure some operators may delay investments and those who invest face a long time from concept definition to commercial equipment that can realize the service. Furthermore, even when these new features are standardized and 
implemented it may not possible to realize them with existing equipment, as even though these can be controlled through standardized interfaces there is little possibility to extend them through the use of open interfaces. In short, further evolution along the same line has drawbacks.

On these grounds, Software-Defined Networking (SDN) and Network Function Virtualization (NFV) emerged. SDN and NFV are not dependent on each other but they are complementary concepts. Ever since its introduction, SDN has gained significant traction in academia and the industry. We refer the interested reader to [1] for a comprehensive survey on the topic. The first generation of SDN deployments is closely associated with campus networks [2], data centres [3] and private backbones [4]. More recently, SDN principles have found their way to carrier-grade architectures such as the 3GPP EPS [5]-[7]. NFV on the other hand rapidly gained considerable momentum from leading network operators which have initiated, together with other operators, IT and equipment vendors and technology providers, a new standards group for the virtualization of network functions [8]. NFV proposes to shift middle box processing from hardware appliances to software running on inexpensive hardware (e.g., x86 servers with $10 \mathrm{~Gb}$ network interface cards) [9]. It involves the implementation of network function in software that can run on general purpose server hardware and that can be instantiated in various locations in the network as required without the need for installation of new equipment [10]. NFV is able to support SDN by providing the infrastructure upon which the SDN software can run. SDN itself, has two defining characteristics. First, the control plane (which decides how to handle traffic) is separated from the data plane (which forwards traffic according to decisions that the control plane makes) in a manner more daring than other carrier-grade architectures, such as the 3GPP Evolved Packet Core (EPC). Second, the control plane is consolidated, so that a single software control program controls multiple data-plane elements (i.e. routers, switches and other middleboxes) via a well-defined API (e.g. OpenFlow) [11]. SDN enables network operators to configure the control of their network through custom software. Both NFV and SDN advocate the use of inexpensive hardware [12] that is software programmable.

The increased softwarization and programmability can help operators to avoid vendor lock-in and makes innovation in network management possible. It allows virtualization of packet based transport networks and allows network providers a greater degree of control over what services they can switch on, where and how quickly [13].

\section{Placeholder for Figure 1}

In this context we advance the state-of-the art by (1) providing a multi-layer modular architecture for carrier networks based on SDN- and NFV principles and (2) by analysing the impact on CapEx and OpEx costs in an IP/MPLS based transport service with SDN capabilities that interconnects the key functional elements in a mobile network.

The remainder of this article is organized as follows. First, we present a brief background on mobile broadband networks today. Second, we sketch the main functional components and layers in the SDN control architecture of a carrier network supporting NFV and we give particular attention to the concept of service function chaining in the context of software-defined mobile networks. Third, we review techno-economic literature and introduce the technical scenarios considered in the cost model. Fourth, we quantify the CapEx and OpEx cost changes for an IP/MPLS based mobile transport network with SDN capabilities. An analysis of key parameters is conducted and we benchmark our results against previous studies. Finally we conclude the article. 


\section{Mobile Broadband Networks Today}

Network operators around the world are currently deploying fourth generation $(4 \mathrm{G})$ networks. The EPS forms the foundation for these networks. It consists of the evolved packet core (EPC), standardized by 3GPP, and the evolved Universal Mobile Telecommunications System (UMTS) terrestrial radio access network (E-UTRAN). The EPC architecture is a closed system based on standardized interfaces where every component typically stands for specialized hardware and software that performs specific operational and management functions such as policing, mobility management, authentication, authorization and accounting, charging, and so on. Figure 2 illustrates the key functional elements involved in typical EPS operations such as music streaming, a voice call or online access to a website. The traffic from the user equipment (UE) is directed from the attached eNB to the serving gateway (SGW) to the packet data network (PDN) gateway (PGW) and onwards to the corresponding PDN. Figure 2 illustrates that the SGW and PGW are user plane elements while the mobility management entity (MME), the home subscriber server (HSS) and the policy control and charging rules function (PCRF) are control plane elements. An IP/Ethernet-based transport network interconnects eNBs with the SGW, PGW and neighbouring eNBs.

\section{Placeholder for Figure 2}

This mobile network architecture is highly optimized and allows network operators to deliver a diverse range of services through secure communications with quality of service guarantees and seamless mobility support to a large numbers of subscribers. However, as new technologies emerge, such as Internet of Things, Cloud Computing and Content Delivery Networks, existing specialized equipment may not be of much use and new specialized hardware, which can be sold after vendors have implemented the new standards, has to be added to the mobile networks. As such due to the high level of specialization and the complex nature of mobile technology, this architecture starts to become a handicap to future growth of the carrier networks.

\section{Software Defined Mobile Broadband Networks}

In order to address the challenges introduced in the previous sections, a multi-layer modular architecture for carrier networks based on SDN- and NFV principles is being explored by the standardization organization European Telecommunications Standards Institute (ETSI) and the research project UNIFY. The network architecture illustrated in Figure 3 is inspired on those projects and on our own previous work [14] and addresses the need to control storage, computing and networking resources in an orchestrated way by decomposing the complex nature of mobile technology into multiple, smaller functional layers each by itself consisting of smaller components. The modularity stimulates maximum component re-usability and enables multiple potential migration paths towards future architectures.

\section{Placeholder for Figure 3}

At the lowest layers, a functional split is introduced between the virtualization layer and the infrastructure layer resources (networking, computing and storage hardware). In the case of server resources, virtualization technology segments one device in multiple logical devices. In the case of networking resources, virtualization technology horizontally slices an entire collection of devices in multiple logically isolated networks. A second 
layer, the control layer interconnects via its southbound interface to the components of the infrastructure layer in order to provide control level services such as a data store services or a topology management service. The highest layer, the application layer, builds further on control layer services to program client applications such as a firewall application or a big data application. Both control architectures are depicted in Figure 3.. The first, on the left side, is in charge of controlling a network of switching and routing equipment. The second, on the right side, is in charge of a network of re-usable compute and storage servers. Orthogonal to the horizontal layers of both architectures, a management layer configures any of the components at the infrastructure-, control- or application layer. An additional layer, the orchestration layer, allows the deployment of services that require a coordinated deployment of both network- and server resources. The architecture allows service- and carrier network operators to offer flexible and dynamic services combining these resources. The realization of such services can be achieved by decomposing the service in service functions. The Internet Engineering Task Force (IETF) describes a service function as a function that is responsible for specific treatment of received packets [15]. The concept of service function chaining is a way of describing the traffic steering that a selection of network traffic must follow in order to cross the necessary network functions. The term chain does not imply that this is a linear sequence as the chain could consist of a complex network of interactions between these components. We do not delve into details with regard to the implementation of this architecture but we would like to refer the interested reader to [16] for a discussion of network service chaining and to [17] for a description of the service function chain of an IP VPN service. We would like to highlight that the EPS can be seen as a service function chain demanding a wellconfigured and interworking packet transport network. In this context we study the economic impact of adding SDN capabilities to a high capacity, virtualized packet transport service.

\section{Techno-economic Analysis Via Cost Modeling}

Techno-economic analysis is typically used to evaluate economic feasibility of a technical solution by utilizing forecasting, network design and investment analysis methods. It can focus on cost modelling, such as this study, but can also be extended to include financial results. The developed model is used to analyse the impact of system parameters on the feasibility of a system solution. This methodology has been widely applied to mobile network scenarios. The authors of [18]-[19] tackle the deployment of universal mobile telecommunications system (UMTS) in conjunction with wireless local area network (LAN) technologies from a techno-economic perspective. The authors of [20] conduct a techno-economic analysis of femtocell deployments in long-term evolution (LTE) networks. In [21], techno-economic modelling has been used to analyse the position of virtual network operators in the mobile communications industry. In [22], a techno-economic approach has been used to identify business alternatives and opportunities for mobile operators. Techno-economic modelling is also frequently used by national regulatory authorities for the evaluation of price setting or policy options in regulated markets [23].

The goal of this study, however, is to quantify the changes in CapEx and OpEx resulting from the application of SDN principles in the transport service of a mobile network as a guidance for strategic decisions. Therefore, cost modelling is applied. This type of modelling is widely used to determine potential cost savings. In [24], for example cost modelling is used to analyse the CapEx savings that can be reached by taking advantage of SDN networks to control a mix of packet and circuit network from a single vantage point. In [25], metrics and formulas are determined for a cost model for cloud computing. In [26], cost modelling is used to evaluate CapEx in optical 
multilayer networks. In most cases (e,g, [24], [26]), an equipment cost model is used to estimate CapEx costs, whereas OpEx costs tend to be neglected or only dealt with summarily, e.g. as a proportion of CapEx. Also, some obscurity exists in the literature concerning the exact definition of CapEx and OpEx. A cost model should therefore have a detailed classification of what type of costs are considered as CapEx and OpEx costs and an explanation of how each of these are quantified. In our previous work [27]-[28], we proposed a classification of CapEx and OpEx costs for network operators which is widely applied in techno-economic research. This classification is also used in the modelling work of this article. In the field of cost modelling for mobile networks, a detailed CapEx and OpEx cost model for LTE networks has been created in the MEVICO project [29]. The considered network architecture does however not involve SDN capabilities. Different studies have been conducted that involve SDN capabilities. In our previous work, we have analysed the impact of software-defined networking as an architecture for the virtualization of mobile networks [30]. The paper focused on quantification of the savings in CapEx in a scenario in which two virtual mobile network operators share the same infrastructure which is enhanced with SDN capabilities.

The focus of the current paper is however different as it considers a single mobile network operator that runs its services over a transport network with SDN capabilities. Another differentiator is that we provide insights in both CapEx and OpEx. OpEx are seldom addressed although they often turn out to be a significant proportion of the total cost of ownership. We therefore include separate OpEx cost calculations, which are not modelled as percentage of the CapEx costs involved, but registered as a different cost that is related to the operational processes that are required to offer mobile service to subscribers via mobile broadband networks. In the remainder of this article, we will assess the economic implications of introducing SDN principles to the transport network of the EPS architecture (SDN scenario). This scenario is benchmarked against a state-of-the-art carrier grade transport network deploying Carrier Ethernet based on IP/MPLS (state-of-the-art, SoTA scenario). Closely related to the work presented in this article, we found two analyst reports [31], [32] and one scientific paper [33]. The network design reported in [31] is similar to our network design and the focus of the analysis is also on the transport network. OpEx are however modelled as a relative percentage of CapEx in this study. The authors of [32] focus on the impact of SDN on the evolved packet core (EPC) of a mobile network. Both CapEx and OpEx are modelled and calculated but the transport network is left out of scope. Finally, in [33] a complete mobile network with SDN capabilities is considered and both CapEx and OpEx are modelled. The study does however not mention OpEx changes for the transport network. At the end of this paper, we benchmark our results against those reported in [31]-[33].

In this article, cost modelling is used to quantify the changes in CapEx and OpEx costs that result from the centralization of control and the operational paradigm of developing and operating the virtual network management software in-house instead of buying a vendor solution only. In-house development may however also increase the risk of service malfunctioning. One possible way to mitigate that risk, is to train and combine development and operations staff in highly skilled DevOps teams. Devops consists of a set of practices to the development of software products and services based on close ties between the departments for development (which writes and tests code) and operations (which operates the virtual infrastructure, network functions and applications). DevOps practices focus on automation, repeatability and predictability of the operational environment, as well as on a close cultural integration between members of various teams. A key component of DevOps is the cultural aspects that create an environment where both departments interact with increased 
efficiency. It enables increased velocity for introducing new services and network functions to operations and the development of customized, operationally optimized software supporting network operations much better than generic products from suppliers. To realize the benefits of DevOps, carrier network providers require an organizational shift in the processes, methods and systems as well as training of staff and organizational change management in order to maintain quality levels like repeatable and reliable processes, advanced monitoring capabilities and feedback loops between operations and development. Major risks are the difficulty to find and retain such staff on the one hand and resistance to organizational change on the other. The successful change from traditional workflows to DevOps driven workflows requires the support from the SP's staff. Therefore both development and operational teams should receive training in the skills involved. These include the processes, methods and systems that have the ability to monitor fault- and performance metrics, to enable ongoing verification of code, to support advance troubleshooting mechanisms and to test against production-like systems.

Similar to [31]-[33], the cost model in this article, does focus on the impact of SDN while it does not take into account the impact of NFV aspects as reliable data points are not available at the time the study was conducted. Also, migration costs (e.g. running two parallel networks for some time) and the costs related to changing the organisational culture (e.g training cost of DevOps teams) are not included in the cost model as these are to a large degree organisation specific.

\section{Evaluation of CaPex and OpEx Costs}

We quantify the CapEx and OpEx cost changes that can be reached in an IP/MPLS-based mobile transport network with SDN capabilities (SDN scenario). This scenario is benchmarked against a state-of-the-art carrier grade mobile transport network with Carrier Ethernet based on IP/MPLS but without SDN capabilities (state-of-the-art, SoTA scenario). Germany is the reference country for this analysis.

\section{Placeholder for Figure 4}

Figure 4 provides and overview of the different steps that will be followed in this analysis. First, we gather input data on traffic sources (number of subscribers and peak traffic demand). Second, both scenarios are translated into a network design. These are inputs to the dimensioning process which provides a bill of resources as output. By combining the bill of resources, cost points and a clear CapEx and OpEx cost classification into a cost model, we are able to compare both scenarios in terms of CapEx and OpEx costs. Each of these steps are detailed below.

\section{TRAFFIC SOURCES}

For this study, the parameters illustrated in Figure 5 are assumed. The number of subscribers and the data per subscribed is composed of a combination of historic data and forecast data of three data sources: (1) Cisco Visual Networking Index [34], (2) data obtained from a mobile operator and (3) data obtained from a network equipment vendor. Some of this data is covered by confidentiality agreements and as such not publicly available. Therefore, average values are published in this article.

Analysis of data concerning the traffic on an eNB revealed traffic demand variation throughout the day, a $7 \%$ traffic share during busy hour is used in the traffic model. In addition, to take into account peak traffic demand during busy hour, we a heavy tailing factor of 3 times the normal demand is used. During analysis of data concerning the traffic per eNB from a large European network operator, a large variation in traffic load between individual eNBs was discovered. The eNB with the highest traffic (top 5\% and 10\%) respectively have around 20 times and 10 times more traffic than an average eNB. There is also a large variation in between the distribution 
of eNBs to their aggregation site. To take this into account, the following mix between types of eNBs to the aggregation site was taken into account: $15 \%$ top 5, 15\% top 10, 70\% normal traffic of respective eNB types. Traffic load per eNB is used as input for the network design.

\section{Placeholder for Figure 5}

\section{NETWORK DESIGN AND DIMENSIONING}

The aim of network dimensioning is to optimize the number of network elements which fulfil the quality-ofservice and capacity requirements for the services offered at the minimal total cost. The network elements are dimensioned based on the carried traffic and the network design. We have carried out market analysis of existing routers and decided for the most common available device types.

For the analysis of the transport network, we design a full mobile network (access, aggregation and core) in order to verify the impact against all elements of a typical network. In the general case, it is assumed that the elements of EPS are not connected directly to each other, but have other network elements interconnecting them. In this study, an access network with 25,000 eNBs and an aggregation network with two aggregation stages has been assumed. Elements are grouped and virtualization technologies are used in order to realize the virtualization benefits like security or simpler configuration of backup paths. The EPS, as schematized in Figure 2, is detailed into a network design in Figure 6. The access network consists of 25,000 eNBs. Each eNB is connected to 1 of the 1,000 pre-aggregation sites with a redundant path to another pre-aggregation site. A preaggregation site is connected to 1 of the 80 aggregation sites with a redundant path to another aggregation site. The aggregation sites are connected to 1 of the 12 core locations with a redundant path to another core site. Of the 12 core sites, 6 are used as inner core in parallel. A combination of mesh and direct connections links the core sites. Each of the 12 core sites is attached redundant to 1 of the 6 inner core sites. By doubling the available capacity at disjunctive locations and appropriate connections a complete redundant network is provided. The inner core is connected to the Internet. We consider 1+1 protection (two connections are set up simultaneously, one of them being used as backup) and the ITU-T G.8032 Ethernet ring protection switching mechanism is used to provide sub-50ms protection and recovery switching for Ethernet traffic in a ring topology. It enables basic virtualization of the packet layer.

A small size router is deployed in both aggregation locations. A medium size router with $40 \mathrm{x} 1 \mathrm{GbE}$ line cards is deployed at each of the core sites that are not an inner core site in parallel and a large size router with a combination of $40 \times 1 \mathrm{GbE}$ and $24 \times 10 \mathrm{GbE}$ line cards is deployed at each of the 6 inner core locations. The $1 \mathrm{GbE}$ links are used to connect to the core platform and in the SDN scenario to the controller. The line cards and transceivers are purchased and installed as needed based on the evolution of the traffic demand. The networking devices require three types of software: an operating system (OS), a license for synchronization support and a VPN license.

To cover the network functions of the EPC we use a core platform that combines the network functions such as the voice and packet gateway function for UMTS, HSPA and LTE in a single specialized platform. The EPC is the same for both scenarios. A performance test for the platform was conducted by [36]. The network design for the EPC is based on these results.

At each location the number of network devices is doubled for redundancy reasons.

\section{Placeholder for Figure 6}




\section{Cost MODEL}

To determine the costs of the dimensioned network, the BoR together with cost data are used to calculate CapEx and Opex. CapEx contribute to the fixed infrastructure and they are depreciated over time. For a network operator, they include the purchase of land, network infrastructure, and software. OpEx do not contribute to the infrastructure; they represent the cost of keeping the company operational and include the cost of technical and commercial operations, administration, etc. As previously stated, the categorization of CapEx and OpEx and the estimation steps required to estimate the costs of realistic network scenarios used in this study are described in [28] and has previously been used for a quantitative analysis of the total cost of a transport network operator in a German reference network [28]. Respectively equations 1 and 2 show how CapEx and Opex costs are calculated.

In general, obtaining an exact prediction of the cost of a mobile network is difficult as a consequence of the many different factors that influence the results. To deal with this complexity, the investment and operating costs assumed in this article are provided by an operator and two equipment vendors (see Table 1). The cost points for network equipment are based on list prices from two network vendors without discounts. The data points for operational costs are a combination of data from data sheets (e.g. floor space and energy consumption) and semistructured interviews with representatives from a network operator. As these data points are covered by confidentiality agreements and is not made publicly available, averaged out values are used in this article.

For CapEx costs, costs exist in the purchase- and installation cost of switches and SDN controllers (i.e. SDN scenario) to realize the transport service. CapEx are reduced in the SDN scenario because the control plane is lifted up from the router and centralized into a SDN controller. With SDN, operators can prevent vendor lock-in and deploy software programmable network switches. Introducing SDN however also involves the introduction of controllers and the development of custom software which accounts for an extra cost.

\begin{tabular}{|c|c|}
\hline & (Eq. 1) \\
\hline $\begin{array}{l}\text { CapEx } \\
=\frac{\text { number of devices }}{\text { site }} \times \text { number of sites } \times \frac{\text { cost }}{\text { device }} \\
+ \text { number of SDN controllers } \times \frac{\text { cost }}{\text { SDN controller }} \\
+ \text { cost of software development } \\
+ \text { cost of first time installation }\end{array}$ & $\begin{array}{l}\text { site components } \\
\text { SDN components } \\
\text { installation cost }\end{array}$ \\
\hline
\end{tabular}

Based on the bill of resources and the cost points of Table 1, CapEx were calculated using equation 1. In an architecture that corresponds to SDN principles, SDN controllers are connected to the core locations. The rather simple and static network design will limit the networking dynamics and decreases performance requirements for a controller. Based on these considerations and expert interviews, a ratio of 1 controller to 100 programmable switches is used. For the SDN scenario two controllers are added to each of the 12 mobile core locations. This assumption is analysed below in the analysis of key parameters section by varying the ratio of SDN controllers to switches. In the SDN scenario, an additional cost is added to the total CapEx cost for the controller and the development cost of control plane software and the applications running on top of the controllers. The cost for the 
controller is based on the price of a state-of-the-art SDN controller. We assume that with SDN, the control plane is captured in software and can be replaced by custom written software. We model this by removing the VPN license from the bill of materials and adding costs for a team of developers. Also, In the SDN scenario, the OS can be simpler as it requires less capabilities, fewer updates and modifications. The cost of the OS is reduced with $25 \%$. An analysis for different values of the reduction in OS cost is added in the analysis of key parameters section (below). The software development cost is modelled as an annual cost of $€ 1.5$ million which is based on semistructured interviews with representatives of one network operator and one network vendor and assumes that highquality open source software is available. To tackle the uncertainty, a comparison for different software development costs is added in the analysis of key parameters section (below). Transmission costs are not considered as they are not expected to change between both of the scenarios. The costs related to installing the equipment are estimated to amount to $13 \%$ of the equipment cost.

For OpEx costs, the continuous cost of infrastructure is calculated from floor space and energy consumption of the dimensioned network equipment. To calculate the cost of floor space, the different geographic situation across sites is taken into account. The cost of power, back-up power and cooling are taken together. The power per device is based on the power required by the router chassis, line cards and the route switch processor. In the SDN scenario, the continuous cost of infrastructure is lower because there is lower energy consumption by the control plane in the network switches. The additional controllers consume less power than what can be saved by centralizing the control plane. The power consumption by the control plane is estimated at $11 \%$ of the total power consumption [35]. The cost of floor space is slightly increased (by the SDN controllers).

The cost of maintenance and repair includes the cost of preventative measures such as monitoring and maintaining the network against possible failures but also the repair of failures in the network. Network care is a process done by the network operations centre (NOC) which is active 24/7. A yearly inventory of software components has to be maintained and on a regular basis upgrades and patches need to be installed. This is an extra task of the NOC for which extra employees are hired and trained. This process is expected to be considerably easier in the SDN scenario because of the centralization of certain software components which are now distributed. Even with careful maintenance occasional failures cannot be avoided. Failures are categorized in two categories: hardware failures and software failures. In case of a hardware failure, broken equipment has to be replaced. In case of software failure, the failure is solvable by a software upgrade, patch or a reboot. Maintenance- and repair cost are lower in the SDN scenario as a single cohesive system is created where in old architectures it was required to manage and maintain a bunch of devices. An example is the maintenance cost of software. Software management is easier because the number of running software versions is reduced to a minimum of one. Similar effects come into play for security management and stock management.

Service provisioning begins with a service request from a potential customer and includes the entire process from order entry by the administration to performing the needed tests, service provisioning, service move or change, and service cessation. Service management is concerned with the process of keeping a service up and running once it has been set up. It includes configuration of new services after the initial rollout and the reconfiguration of existing services. Cost of service provisioning and service management is lower because SDN enables automated configuration of the network. For the considered scenarios, there is no direct contact with the customer. A service is therefore defined as a configuration of a transport link between two locations. Service management includes the configuration of the connection and the documentation. This process is expected to be 
considerably easier with the SDN approach because a higher level of automated configuration is possible. Based on structured interviews with staff from the NOC, this was modelled by reducing the time spent at the operational steps of the service provisioning and management process.

Note that we did not include general OpEx parts (up-front planning, customer relationship management, non-telco-specific continuous cost of infrastructure and non-telco-specific administration) as they are common to both scenarios.

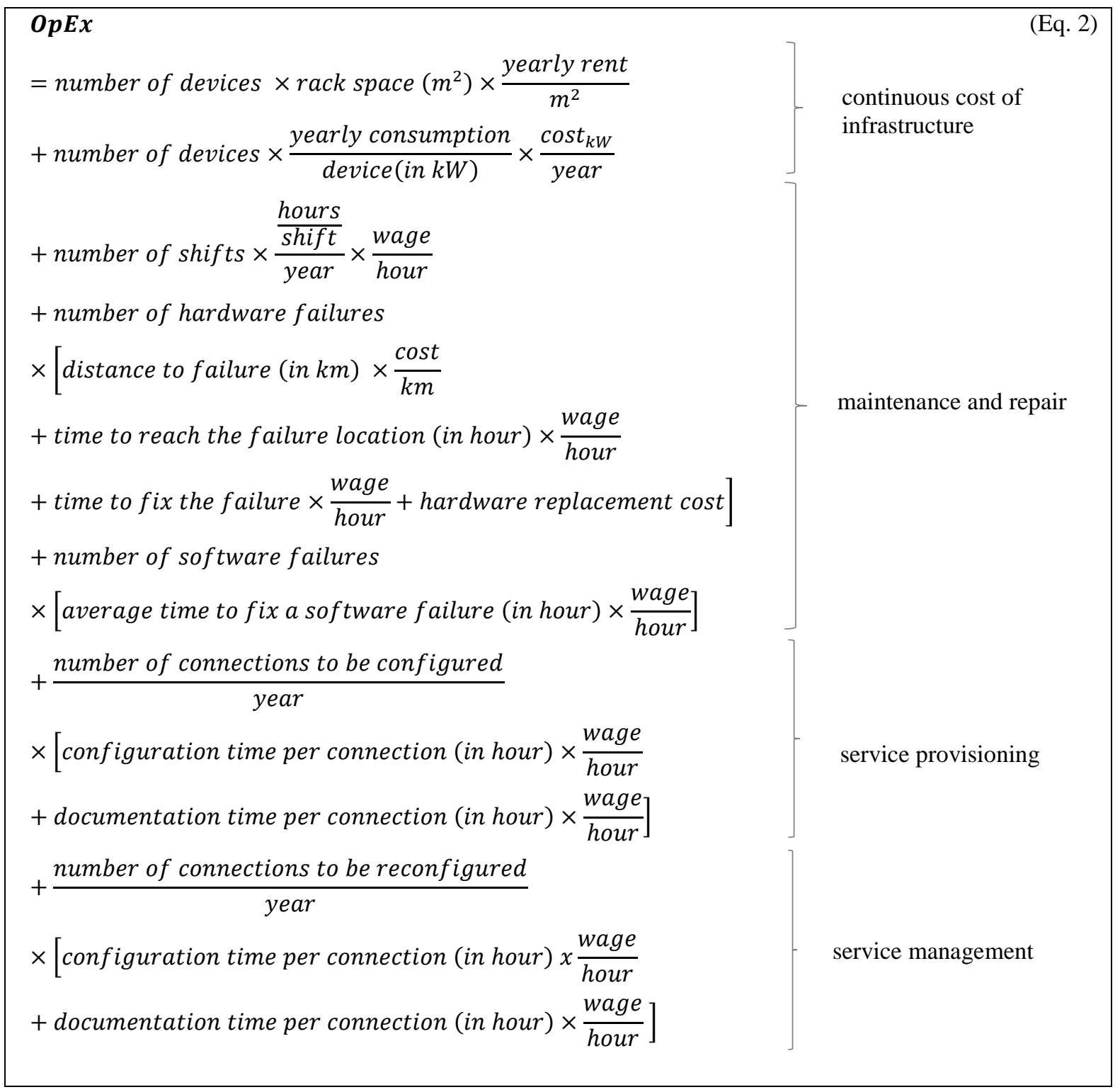




\section{SYSTEM COMPARISON}

The last step of the model is the comparison of the different system solutions. The benefits and drawbacks for each of the scenarios are summarized in Table 2.

\section{Placeholder for Table 2}

The result of network dimensioning and the cost model have been combined in order to obtain the CapEx and OpEx costs over a time period of six years (2012-2017). The network equipment is fully depreciated over this period. Table 1 benchmarks the SDN scenario against the SoTA scenario. Note that the table rows represent per category total costs over the six year period.

The savings for the SDN scenario are $12 \%$. CapEx and OpEx savings are respectively $65 \%$ and $35 \%$ of the total savings.

\section{Placeholder for Table 3}

\section{ANALYSIS OF KEY PARAMETERS}

An analysis of key parameters is used to tackle the uncertainty in the output of the CapEx and OpEx cost model which is a result of the uncertainties of the estimated input parameters. For key parameters, the input parameters are varied and the impact is reported.

The results of this analysis are summarized in Table 4.

\section{Placeholder for Table 4}

In the cost model, $1 \mathrm{SDN}$ controllers is assumed to be able to steer 100 network elements. The ratio was varied between 50 and 200. The analysis shows that the ratio of network elements that a SDN controller is able to steer has a small impact on both CapEx and OpEx costs. This is explained by the relative low price, energy consumption and footprint of an SDN controller compared to the total CapEx.

In our analysis the VPN license of the routers is replaced by software that is developed in-house as well as part of the router's OS. In the cost model, an annual cost of $€ 1.5$ million is used for the cost of in-house software development. However, predicting the effort required to develop software is often hard resulting in uncertain estimates. As such we vary the annual software development cost and report the maximum annual cost before the SDN approach no longer has cost advantages compared to the SoTA scenario. With an annual development cost of $€ 11$ million (7.3 times the original budget), the SDN approach is no longer beneficial.

In the cost model, the operating system's cost is reduced by $25 \%$ in the SDN scenario. However, finding a less complex OS that is compatible with the network elements may not be possible. When the OS cost is kept at its original price, the CapEx cost savings are reduced from $12.04 \%$ to $9.68 \%$. For this case, the maximum annual budget for software development may not exceed $€ 10$ million before the SDN approach is no longer more cost efficient.

The cost points for equipment were derived from the official price list of equipment vendors. Network operators typically negotiate considerable wholesale price discounts. We simulated discount rates of up to $50 \%$ in steps of $12.50 \%$. For higher discount rates, the advantage of the SDN scenario over the SoTA scenario is reduced for CapEx while the SDN advantage increases for OpEx. For CapEx, the reduction can be explained by the combination of two factors. First, the discount applies to all components bought including software licenses which decreases the absolute benefit for the SDN scenario (in which software licenses are partially replaced by software that is developed in-house). Second, in the SDN scenario, for the in-house development of software the development cost cannot be reduced. For OpEx, the higher relative cost savings are a result of the lower proportion 
of operational processes that have low cost savings in the total costs. Maintenance and repair for example becomes less costly in absolute terms (cost of spare parts is lower) while the cost benefit for the SDN scenario is relatively low for these operational processes compared to others such as service provisioning and service management of which the absolute cost does not change.

\section{RELATED WORK}

Our work is based on a set of assumptions and input values that are gathered from network operators and network equipment vendors. The estimation of these input values is often a point of discussion. To cope with this uncertainty we benchmark our results against two studies commissioned by network equipment vendors that compare similar scenarios.

The mobile backhaul network designed in [31] is comparable to the network designed in this article. The SDN scenario has $80 \%$ lower CapEx and 79\% lower OpEx. The results are highly diverging from our results. We traced this divergence to two main reasons: (1) oversimplified devices in the SDN scenario in contrast to the base scenario in which state-of-the-art IP/MPLS routers are dimensioned. This enlarges the gap between both scenarios and in particular for CapEx. (2) For OpEx, the results are highly correlated with CapEx, therefore for both categories of expenditures almost the same, high level of savings has been found.

The authors of [33] analyse the cost efficiency of a software-defined LTE-based mobile network via a case study for a Finnish reference network. CapEx and OpEx are modelled (largely) independent from each other. The quantitative results show that SDN reduces the network related annual CapEx by $7.72 \%$ and OpEx by $0.31 \%$ compared to a non-software-defined LTE-based mobile network. These costs savings are an order of magnitude lower than the ones reported in [31] and also lower than our results. The divergence can be traced back to two factors. First, in contrast to our work, the authors of [33] include the investment expenses of equipment for the eNBs and EPCs while our study focuses solely on the backhaul network. As the authors of [33] report a cost increase of $14.11 \%$ for the EPC and a cost reduction of 7.15 for the eNB the total CapEx cost savings are impacted by these. When zooming in to the CapEx cost savings that can be reached for the transport network, our results are similar to the reported results in [33], $12.04 \%$ and $13.50 \%$ cost savings respectively. Second, for OpEx, the authors of [33] use a different classification for operational expenses which makes comparison hard. Also the calculation steps are not detailed. However, focusing on energy consumption we report a net decrease in energy consumption as we expect that part of the control functions can be turned off on the switch and performed by a centralized controller which is able to control multiple switches at once. This contrasts with the results reported in [33] who expect an increase by $0.07 \%$ in energy consumption due to the addition of SDN controllers and no ability to switch off part of the control functions in the switch. Also energy consumption is by far the largest cost in the OpEx model of [33] as large savings in all other operational processes (e.g. $29.32 \%$ in network management) are hardly able to offset the small increase in energy cost. In our model, operational processes related to network management (e.g. service provisioning and service management) represent a higher share of total costs. As we, similar to the authors of [33], expect substantial cost savings in these operational processes and their weight in the total cost is higher, our total cost savings are also higher.

The authors of [32] focus on the move of the EPC core network functions to the cloud. This study focuses on a different part of the network but the same drivers are at play. The study reports saving up to $20 \%$ over 5 years compared to a traditional EPC architecture. CapEx and OpEx savings are respectively $72 \%$ and $28 \%$ of the total savings. The two main cost drivers are the network elements and software functions (CapEx) and the cost of 
staffing (OpEx). These results are in line with our results. The CapEx savings are a result of the use of standardized hardware and middleware and greater economies of scale. The OpEx savings are a result of closer integration between the core network and the network management which results in simplified service provisioning and management. Similar to our findings, the savings in site rental, power consumption and maintenance and repair are relatively insignificant. The divergence in total cost savings is traced back to higher savings in CapEx. However, it should be noted that the authors themselves challenge the extend of savings that can be derived from the network node and software functions.

\section{Conclusion}

We introduced a multi-layer modular architecture for carrier networks based on SDN- and NFV principles and conducted a techno-economic evaluation that considers application of SDN principles to the transport network of the EPS. A detailed CapEx and OpEx cost model as well as input values are described. The changes in both CapEx and OpEx costs were quantified in a case study. CapEx costs are reduced in the SDN scenario because the control plane is lifted up from the router and centralized into a controller and the cost of software licenses is reduced. The main difference in OpEx cost can be found in the cost of service provisioning and management due to the possibility to reduce the amount of manual configuration required and better testing abilities ahead of service rollout. For the case study, the savings for the SDN scenario are quantified at $12 \%$ compared to the SoTA scenario. CapEx and OpEx savings are respectively $65 \%$ and $35 \%$ of total savings.

We expect that cost containment and simplification of the network are the minimum requirements for the SDN approach to succeed but that they are not sufficient to spur widespread adoption of SDN by mobile network operators in their own right. Hence, widespread adoption will only succeed if the mobile network can be opened to support further innovation inside their network without having to depend on equipment vendors to support their innovation.

\section{ACKNOWLEDGEMENTS}

This work has been partially funded by the European Commission under the 7th Framework research programme projects SPARC and UNIFY.

\section{REFERENCES}

[1] Kreutz D. et al., "Software-defined networking: A comprehensive survey," Proceedings of the IEEE, vol. 103, no 1, 2015, pp.14-76

[2] McKeown N. et al., "OpenFlow: enabling innovation in campus networks," ACM SIGCOMM CCR, vol.38, no.2, ACM, 2008, pp. 69-74.

[3] Nicira, "It's time to virtualize the network," 2012

[4] Jain S. et al. "Experience with a globally deployed software defined WAN network-virtualizationplatform," Proc. ACM SIGCOMM, Hong Kong, China, 2013.

[5] Li E. et al., "Toward Software-Defined Cellular Networks," Proceedings of the European Workshop on Software-Defined Networking," 2012, pp. 7-12 
[6] Kempf J. et al., "Moving the mobile evolved packet core to the cloud," Proceedings of the IEEE International Conference on Mobile Computing, Networking and Communications, 2012, pp. 784-791

[7] Pentikousis K. et al., "Mobileflow: Toward software-defined mobile networks," IEEE Communications Magazine., vol. 51, no.7, 2013, pp.44-53

[8] ETSI, "Leading operators create ETSI standards group for network functions virtualization," http://www.etsi.org/news-events/news/644-2013-01-isg-nfv-created, 2013

[9] Martins J. et al., "ClickOS and the art of network function virtualization", Proceedings of the $11^{\text {th }}$ USENIX Symposium on Networked Systems Design and Implementation, 2014, pp. 459-476

[10]ETSI, "Network Functions Virtualisation: An Introduction, Benefits, Enablers, Challenges and Call for Action”, https://portal.etsi.org/NFV/NFV_White_Paper.pdf, 2012

[11]Feamster N. et al., "The road to SDN: an intellectual history of programmable networks," ACM QUEUE, vol.11, no.12, ACM, 2013

[12] Greenhalgh A. et al., "Flow processing and the rise of commodity network hardware," ACM SIGCOMM CCR, vol. 39, no.2, ACM, 2009, pp. 20-26

[13] Alshaer H., “An overview of network virtualization and cloud network as a service,” International Journal of Network Management, vol. 25, no 1, 2015, pp 1-30

[14] Tavernier W. et al., "Can open-source projects (re-) shape the SDN/NFV-driven telecommunications market?,"it-Information Technology, vol. 57, no. 5, 2015, pp.267-276

[15] Quinn P. and Nadeau T., "Service Function Chaining Problem Statement,” Internet-Draft draft-ietf-sfcproblem-statement-10.txt, IETF Secretariat, Aug. 11, 2014

[16] John W. et al. "Research directions in network service chaining," Proc. Software Defined Networking for Future Networks and Services (SDN4FNS), 2013

[17] Kim J. et al., "Service provider DevOps for large scale modern network services," Proc. 10 ${ }^{\text {th }}$ International Workshop on Business-driven IT Management, part of IM2015, the IEEE International Symposium on Integrated Network Management, 2015, pp.1391-1397

[18] Mitjana E. et al., "Seamless IP service provision: techno-economic study by the MIND and TONIC projects," Proceedings of the IST-Information Society Technologies, 2002

[19] Harno J., "Techno-economic analysis of beyond 3G mobile technology alternatives," info, vol. 11, no.3, 2009, pp. 45-63

[20] Frias Z. et al., "Techno-economic analysis of femtocell deployment in long-term evolution networks," EURASIP journal on wireless communications and networking, 2012, pp. 1-15

[21] Smura T. et al., "Virtual operators in the mobile industry: a techno-economic analysis," NETNOMICS: Economic Research and Electronic Networking, vol. 8, no. 1-2, 2007, pp. 25-48

[22] Harno J. et al., "Alternatives for mobile operators in the competitive $3 \mathrm{G}$ and beyond business", Telecommunication Systems, vol. 42, no. 2, 2009, pp. 77-95

[23] Ofcom, "Application of spectrum liberalisation and trading to the mobile sector. A further consultation" Consultation document, http://stakeholders.ofcom.org.uk/consultations/spectrumlib/, 2009

[24] Das S. et al., "Rethinking IP Core Networks," Journal of Optical Communications and Networking, vol. 5 no.12, 2013, pp. 1431-1442 
[25] Li X. et al., "The Method and tool of cost analysis for cloud computing," Proceedings of the IEEE Conference on Cloud Computing, 2009, pp. 93-100

[26] Huelsermann R. et al., "Cost modeling and evaluation of capital expenditures in optical multilayer networks," Journal on Optical Networking, vol. 7, no. 9, pp. 814-833

[27] Verbrugge S. et al., "Modeling operational expenditures for telecom operators," Proceedings of the Conference on Optical Network Design and Modelling, 2005, pp. 455-466

[28] Verbrugge S. et al., "Methodology and input availability parameters for calculating OpEx and CapEx costs for realistic network scenarios," Journal of Optical Networking, vol. 5, no. 6, 2006, pp. 509-520

[29] MEVICO, “Mobile Networks Evolution for Individual Communications Experience” EU Celtic project", 2011-2014

[30] Naudts B. et al., "Techno-economic analysis of software-defined networking as architecture for the virtualization of a mobile network", Proceedings of the European Workshop on Software Defined Networking, 2012, pp. 67-72

[31] ACG Research, “A TCO analysis of Ericsson's virtual network system concept applied to mobile backhaul," 2012

[32] PA Consulting Group, "EPC and Intel's vision for the telco cloud,” 2012

[33]Zhang N. et al., "Cost efficiency of SDN in LTE-based mobile networks: Case Finland" Proceeding of the IEEE International Conference and Workshop on Networked Systems, 2015, pp.1-15

[34] Cisco, Cisco Visual Networking Index. "Global mobile data traffic forecast update, 2013-2018." White paper, 2014

[35] Tucker R. et al., "Evolution of WDM optical IP networks: A cost and energy perspective," IEEE JLT., vol. 27, no. 3, 2009

[36] Rossenhövel C. et al., “Testing Cisco’s mobile core, data center \& business services,” EANTC, 2011. 
Table 1. Network element configurations and per-unit cost assumptions

\begin{tabular}{|c|c|c|c|}
\hline \multicolumn{4}{|c|}{ general parameters } \\
\hline & & & $\operatorname{cost}(€)$ \\
\hline \multicolumn{3}{|c|}{ hourly wage of employee in customer service } & 45.00 \\
\hline \multicolumn{3}{|c|}{ hourly wage of employee in network operations center } & 58.00 \\
\hline \multicolumn{3}{|l|}{ hourly wage of field technician } & 52.00 \\
\hline \multicolumn{3}{|l|}{ yearly price per $\mathrm{kW}$} & $2,700.00$ \\
\hline \multicolumn{4}{|c|}{ network elements } \\
\hline & $\operatorname{Price}(€)$ & $\begin{array}{c}\text { power } \\
\text { consumption(W) }\end{array}$ & MTBF (hours) \\
\hline $\begin{array}{l}\text { small size router } \\
\text { ( } 4 \text { integrated } 10 \mathrm{GbE} \text { SFP ports) }\end{array}$ & $44,000.00$ & 335 & $175,200.00$ \\
\hline medium size router & $35,000.00$ & 610 & $175,200.00$ \\
\hline large size router & $37,000.00$ & 835 & $175,200.00$ \\
\hline router OS & $13,000.00$ & - & $110,000.00$ \\
\hline VPN license & $17,000.00$ & - & $110,000.00$ \\
\hline IEEE 1588 support & $11,000.00$ & - & $110,000.00$ \\
\hline Line card $20 \times 1 \mathrm{GbE}$ & $8,500.00$ & 420 & $110,000.00$ \\
\hline Line card $40 \times 1 \mathrm{GbE}$ & $21,000.00$ & 350 & $110,000.00$ \\
\hline Line card $24 \times 10 \mathrm{GbE}$ & $147,000.00$ & 895 & $110,000.00$ \\
\hline 1000BASE-SX MMF (550m) & 500.00 & - & $300,000.00$ \\
\hline 1000BASE-ZX SMF(70km) & $3,500.00$ & - & $300,000.00$ \\
\hline 10GBASE-SX MMF(550m) & $1,300.00$ & - & $300,000.00$ \\
\hline 10GBASE-ZX SMF(70km) & $12,500.00$ & - & $300,000.00$ \\
\hline SDN controller & $50,500.00$ & 660 & $175,200.00$ \\
\hline $\begin{array}{l}\text { annual cost of in-house software } \\
\text { development }\end{array}$ & $1,500,000, .00$ & & \\
\hline \multicolumn{4}{|c|}{ cost of floor space } \\
\hline & & & Value \\
\hline \multicolumn{3}{|c|}{ ratio urban/dense urban (pre-)aggregation sites(\%) } & $15 / 85$ \\
\hline \multicolumn{3}{|l|}{ ratio urban/dense urban core sites(\%) } & $0 / 100$ \\
\hline \multicolumn{3}{|l|}{ yearly rent urban(euro) } & 170 \\
\hline \multicolumn{3}{|l|}{ yearly rent dense urban(euro) } & 220 \\
\hline
\end{tabular}




\begin{tabular}{|c|c|c|}
\hline \multicolumn{2}{|l|}{ correction factor } & 2.65 \\
\hline \multicolumn{2}{|l|}{ rack space $\left(\mathrm{m}^{2}\right)$} & 0.78 \\
\hline \multicolumn{3}{|c|}{ cost of repair } \\
\hline & & Value \\
\hline \multicolumn{2}{|l|}{ distance to the failure (kilometer) } & 100.00 \\
\hline \multicolumn{2}{|l|}{ cost per km (euro) } & 0.40 \\
\hline \multicolumn{2}{|l|}{ time to reach the failure location (hours) } & 1.00 \\
\hline \multicolumn{2}{|l|}{ time to fix failure (hours) } & 3.00 \\
\hline \multicolumn{2}{|l|}{ hardware replacement cost (euro) } & cost of component \\
\hline \multicolumn{2}{|l|}{ time to fix software failure (hours) } & 2.00 \\
\hline \multicolumn{3}{|c|}{ cost of network care } \\
\hline \multicolumn{3}{|c|}{\begin{tabular}{|l|l|} 
& Value \\
&
\end{tabular}} \\
\hline \multicolumn{2}{|l|}{ number of shifts } & 5.00 \\
\hline \multicolumn{2}{|l|}{ employees per shift (number of FTEs) } & 10.00 \\
\hline \multicolumn{3}{|c|}{ service provisioning and management } \\
\hline & Time (in hours) & time SDN (hours) \\
\hline service planning and management & 0.80 & 0.50 \\
\hline network planning & 2.25 & 1.00 \\
\hline service accounting and administration & 0.80 & 0.80 \\
\hline
\end{tabular}

Table 2. Comparison of benefits and drawbacks of each scenario

\begin{tabular}{|c|c|c|}
\hline & Benefits & Drawbacks \\
\hline \multirow[t]{2}{*}{ SoTA } & $\begin{array}{l}\text { Well-known, proven and highly successful } \\
\text { way of doing business. }\end{array}$ & $\begin{array}{l}\text { Complex to manage existing services. Low } \\
\text { flexibility to introduce new services. }\end{array}$ \\
\hline & Proven technology with mature suppliers. & $\begin{array}{l}\text { Increasing CapEx and OpEx versus stable } \\
\text { ARPU. }\end{array}$ \\
\hline \multirow[t]{2}{*}{ SDN } & $\begin{array}{l}\text { Promise of higher service flexibility and easier } \\
\text { service management. }\end{array}$ & $\begin{array}{l}\text { Disruptive technology has increased risk of } \\
\text { start-up problems. For example, open-source } \\
\text { projects may not be stable. }\end{array}$ \\
\hline & $\begin{array}{l}\text { Lower CapEx and OpEx. SDN is } \\
\text { complementary to NFV, possibly reducing } \\
\text { costs further. }\end{array}$ & $\begin{array}{l}\text { Organizational change can be hard to manage. } \\
\text { For example the transition towards in-house } \\
\text { software development may suffer from staff } \\
\text { resistance. }\end{array}$ \\
\hline
\end{tabular}

Table 3. Total CapEx and OpEx costs for German reference scenario in the period 2012-2017 


\begin{tabular}{|c|c|c|c|c|}
\hline & CapEx/OpEx category & SoTA scenario $(€)$ & SDN scenario $(€)$ & Delta (\%) \\
\hline \multirow[t]{5}{*}{ CapEx } & Pre-aggregation sites & $227,224,806$ & $186,914,729$ & -17.74 \\
\hline & Aggregation sites & $33,996,899$ & $30,772,093$ & -9.49 \\
\hline & Core sites & $27,553,953$ & $25,461,302$ & -7.59 \\
\hline & SDN components & / & $10,581,581$ & \\
\hline & First time installation & $37,540,837$ & $33,314,267$ & -11.26 \\
\hline \multicolumn{2}{|r|}{ Total CapEx } & $326,316,495$ & $287,043,973$ & -12.04 \\
\hline \multirow[t]{4}{*}{ OpEx } & $\begin{array}{l}\text { Continuous cost of } \\
\text { infrastructure }\end{array}$ & $38,647,668$ & $36,557,136$ & -5.41 \\
\hline & Maintenance and repair & $90,687,586$ & $90,015,846$ & -0.74 \\
\hline & Service management & $26,890,760$ & $15,553,473$ & -42.16 \\
\hline & Service provisioning & $14,506,367$ & $8,389,707$ & -42.17 \\
\hline \multicolumn{2}{|r|}{ Total OpEx } & $170,732,382$ & $150,516,162$ & -11.84 \\
\hline \multicolumn{2}{|r|}{ Total } & $497,048,877$ & $437,560,135$ & -11.97 \\
\hline
\end{tabular}

Table 4. Results of the analysis of key parameters

\begin{tabular}{|l|c|c|c|c|c|}
\hline Ratio of SDN controllers to network elements & $1: 50$ & $1: 75$ & $\mathbf{1 : 1 0 0}$ & $1: 150$ & $1: 200$ \\
CapEx cost change & $-11.17 \%$ & $-11.82 \%$ & $\mathbf{- 1 2 . 0 4 \%}$ & $-12.25 \%$ & $-12.47 \%$ \\
OpEx cost change & $-11.21 \%$ & $-11.68 \%$ & $\mathbf{- 1 1 . 8 4 \%}$ & $-12.00 \%$ & $-12.15 \%$ \\
Total cost change & $-11.18 \%$ & $-11.77 \%$ & $\mathbf{- 1 1 . 9 7 \%}$ & $-12.16 \%$ & $-12.36 \%$ \\
\hline Annual cost of software development & $\mathbf{1 . 5} \mathbf{m i o}$ & 3 mio & 6 mio & 11 mio & 12 mio \\
CapEx cost change & $\mathbf{- 1 2 . 0 4 \%}$ & $-9.20 \%$ & $-3.54 \%$ & $5.90 \%$ & $7.79 \%$ \\
OpEx cost change & $\mathbf{- 1 1 . 8 4 \%}$ & $-11.84 \%$ & $-11.84 \%$ & $-11.84 \%$ & $-11.84 \%$ \\
Total cost change & $\mathbf{- 1 1 . 9 7 \%}$ & $-10.11 \%$ & $-6.39 \%$ & $-0.19 \%$ & $1.05 \%$ \\
\hline Cost reduction of router OS & $0 \%$ & $10 \%$ & $\mathbf{2 5 \%}$ & $40 \%$ & $50 \%$ \\
CapEx cost change & $-9.68 \%$ & $-10.86 \%$ & $\mathbf{- 1 2 . 0 4 \%}$ & $-13.74 \%$ & $-14.75 \%$ \\
OpEx cost change & $-11.84 \%$ & $-11.84 \%$ & $\mathbf{- 1 1 . 8 4 \%}$ & $-11.84 \%$ & $-11.84 \%$ \\
Total cost change & $-10.42 \%$ & $-11.19 \%$ & $\mathbf{- 1 1 . 9 7 \%}$ & $-13.09 \%$ & $-13.75 \%$ \\
\hline Wholesale price discount & $\mathbf{0 \%}$ & $10 \%$ & $20 \%$ & $30 \%$ & $50 \%$ \\
CapEx cost change & $\mathbf{- 1 2 . 0 4 \%}$ & $-11.72 \%$ & $-11.33 \%$ & $-10.82 \%$ & $-9.20 \%$ \\
OpEx cost change & $\mathbf{- 1 1 . 8 4 \%}$ & $-12.23 \%$ & $-12.64 \%$ & $-13.07 \%$ & $-14.02 \%$ \\
Total cost change & $\mathbf{- 1 1 . 9 7 \%}$ & $-11.90 \%$ & $-11.83 \%$ & $-11.74 \%$ & $-11.49 \%$ \\
\hline
\end{tabular}


Bram Naudts received an MSc degree in applied economics: business engineering from Ghent University (Ghent, Belgium) in 2011. He joined the same university in 2011, where he joined the IBCN group of the INTEC department at the Faculty of Engineering and Architecture. His current research focuses on techno-economic evaluation of communication network architectures and services. Recently he is involved in the projects ICT SPARC, ICT UNIFY and ICT FLAMINGO.

Mario Kind received a Dipl.-Inf (FH) in communications and information technology at University of Applied Sciences Leipzig. He is an expert in the area of broadband access networks and network architecture evolution. His focus is on economic evaluation of business, technology and service trends in the telecommunication industry. $\mathrm{He}$ is author or co-author of several contributions international conferences and journals. He was involved in projects like IST 4WARD, IST MobiThin and CELTIC ECOSYS. Recently he is involved in the projects ICT SPARC and ICT OFELIA and is currently involved in the ICT UNIFY project.

Sofie Verbrugge received an MSc degree in computer science engineering from Ghent University (Ghent, Belgium) in 2001. She obtained the $\mathrm{PhD}$ degree from the same university in 2007 for her thesis entitled "Strategic planning of optical telecommunication networks in a dynamic and uncertain environment". Since 2008, she has been working as a researcher affiliated to iMinds, where she is a coordinator for the techno-economic research within the Internet Based Communication Networks and Services group (IBCN). Since October 2014 she is appointed as part-time professor in the field of techno-economics at Ghent University. Her main research interests include infrastructure as well as operational cost modeling, telecom service and network deployment planning, advanced evaluation techniques including real options and game theory.

Didier Colle is full professor at Ghent University since 2014. He was associated professor since 2011 at the same university and received a PhD degree in 2002 and a M. Sc. Degree in electrotechnical engineering in 1997 from the same university. He is group leader in the iMinds Internet Technologies department. He is co-responsible for the research cluster on network modelling, design and evaluation (NetMoDeL) inside the Internet Based Communication Networks and Services (IBCN) research group. This research cluster deals with fixed internet architectures and optical networks, green-ict, design of network algorithms and techno-economic studies.

Mario Pickavet is professor at Ghent University since 2000 where he is teaching courses on discrete mathematics, broadband networks and network modeling. He is leading the research cluster on Network Design, Modeling and Evaluation, together with Prof. Didier Colle. In this context, he is involved in a large number of European and national research projects, as well as in the Technical Programme Committee of a dozen of international conferences. 